\title{
Exacerbations and associated healthcare cost in patients with COPD in general practice
}

\author{
T.R.J. Schermer ${ }^{1}$, C.G.J. Saris 1 , W.J.H.M. van den Bosch1, N.H. Chavannes², \\ C.P. van Schayck², P.N.R. Dekhuijzen³, C. van Weel ${ }^{1}$
}

ABSTRACT: Exacerbations and associated healthcare cost in patients with COPD in general practice. T.R.J. Schermer, C.G.J. Saris, W.J.H.M. van den Bosch, N.H. Chavannes, C.P. van Schayck, P.N.R. Dekhuijzen, C. van Weel.

Background. Acute exacerbations are a characteristic clinical expression of chronic obstructive pulmonary disease (COPD). The objective of this study was to investigate the occurrence rate, management, and healthcare costs of exacerbations in patients with COPD in Dutch general practice.

Methods. Baseline data set from the COPD on Primary Care Treatment (COOPT) trial was used. Details on the occurrence and management of exacerbations were collected by systematic medical record review for the 2-year period preceding trial inclusion.

Results. The mean age of the 286 study subjects involved was 59.2 (SD 9.6) years, postbronchodilator FEV
67.1\% (SD 16.2) of predicted. Following ERS criteria, subjects suffered from: no (26\%); mild (19\%); moderate $(40 \%)$; or severe $(15 \%)$ airflow obstruction. The overall mean and median annual exacerbation rates were 0.88 (SD 0.79) and 0.5 (IQR 1.0), respectively. Exacerbation rate was not related to severity of airflow obstruction $(\mathrm{p}=\mathbf{0 . 6 2 8})$. Mean annual exacerbation costs per subject were $€ 40$, $€ 53$, $€ 61$ and $€ 92$ for the respective severity subgroups $(p=0.012)$. The increase of costs in the more severe subgroups was mainly attributable to more physician consultations, diagnostic procedures, and prescription of reliever medication (e.g., bronchodilators, cough preparations).

Conclusions. Occurrence of exacerbations did not depend on the severity of airflow obstruction, whereas the healthcare cost associated with exacerbations increased along with the severity of the disease.

Monaldi Arch Chest Dis 2006; 65: 3, 133-140.

Keywords: Pulmonary disease, chronic obstructive, exacerbation, health care costs, general practice.

1 Department of General Practice, Radboud University Nijmegen Medical Centre,

2 Department of General Practice, University of Maastricht,

${ }^{3}$ Department of Chest Diseases, Radboud University Nijmegen Medical Centre, The Netherlands.

Correspondence: Tjard RJ Schermer, PhD; Radboud University Nijmegen Medical Centre, Department of General Practice (117/HAG); P.O. Box 9101; 6500 HB Nijmegen, The Netherlands; e-mail: T.Schermer@hag.umcn.nl

This study was funded by the Dutch Council for Health Insurances and the Netherlands Asthma Foundation.

\section{Introduction}

Chronic obstructive pulmonary disease (COPD) is one of the leading causes of morbidity and mortality world-wide [1]. In Western countries, the burden of COPD on society is expected to increase substantially over the next decades $[2,3]$. Apart from the accelerated decline in lung function, an important and characteristic clinical feature of COPD is the occurrence of exacerbations, i.e. recurrent episodes of acute deterioration of the disease caused by infectious agents or other trigger factors [4]. Exacerbations have been shown to contribute substantially to the impaired health status observed in patients with COPD [5-7]. Moreover, several studies suggest that the occurrence of exacerbations may influence the long-term prognosis in terms of lung function decline, health status, and survival [8-11]. Published reports indicate that there may be a large variation in the occurrence rate of exacerbations among patients with COPD. Estimates range from one to three exacerbations per patient per year [12-18], although there is evidence of substantial underreporting by patients [14]. Differences in the populations studied and the absence of a definition for exacerbations generally agreed upon hampers direct comparison of studies.

Acute exacerbations are responsible for a large proportion of the healthcare costs due to COPD and disease severity seems to be the major driver of costs, especially while expenditures for physician time, emergency room visits, hospital admissions, oxygen therapy, and nursing home stays increase as the disease becomes more severe [1922]. However, most published studies have recruited patients in secondary care settings and, consequently, have predominantly included patients suffering from (very) severe COPD. This subgroup of patients, which comprises only a relatively small proportion of all patients with COPD in the general population [23], suffer from frequent and severe exacerbations and are responsible for a substantial part of the healthcare cost attributable to COPD. 
Patients with mild to moderate disease, which are much larger in number [23], are typically treated in primary care and are less well studied with regard to their exacerbations.

Therefore, the objectives of the current study were to investigate the occurrence rate and management of acute exacerbations in patients with COPD treated in Dutch general practices, and to assess the exacerbation related healthcare costs in different stages of severity of airflow obstruction.

\section{Methods}

\section{Study subjects and design}

The study consisted of a systematic medical record review over the past two years in $286 \mathrm{sub}-$ jects with physician-diagnosed COPD recruited from 44 general practices in the Netherlands. All subjects participated in the COPD on Primary Care Treatment (COOPT) study, a randomised controlled trial evaluating the effectiveness of $N$ acetylcysteine and fluticasone propionate in a primary care COPD population [24]. General practitioners (GPs) identified subjects with a diagnosis of COPD using existing diagnostic labels (i.e., International Classifications of Primary Care (ICPC) codings R91 (chronic bronchitis) and R95 (COPD/ emphysema) [25] and drug prescription records (i.e. prescriptions for anticholinergics and inhaled corticosteroids in the previous year). Subjects identified as suffering from COPD were invited for a screening visit to the general practice. Subsequently, eligibility for trial participation was verified in a certified pulmonary function laboratory. Trial inclusion criteria were: aged $35-75$ yrs; current or ex-smoker; chronic dyspnea, sputum production and cough for at least three consecutive months per year during the previous two years [26]; either post-bronchodilator (BD) $\mathrm{FEV}_{1}$ (forced expiratory volume in one second) $<90 \%$ of the predicted value or post-BD $\mathrm{FEV}_{1} / \mathrm{FVC}<88 \%$ ( $89 \%$ for women) of the predicted value; no previous treatment for asthma, allergic rhinitis or atopic rash; no severe comorbid conditions. Subjects with $\mathrm{FEV}_{1}<40 \%$ of predicted post-BD were excluded. The study was approved by the medical ethics review board of the Radboud University Nymegen Medical Centre, the Netherlands. All subjects gave their written informed consent.

\section{Data collection}

GPs identified all contacts related to acute deterioration of the respiratory condition during the two years preceding the date of trial inclusion from the subjects' medical record using a standardised data extraction form. For each study subject the exact time frame was individually marked out by providing the GP with the begin and end dates of the observation period. Required details for each contact were: date, time, and type of contact (office consultation, telephone consultation, home visit); changes in respiratory symptoms (i.e., increased dyspnea, cough, amount of sputum); ob- jective signs (sputum color, sputum consistency, fever); drugs prescribed (dosage and brand of oral glucocorticoids, antibiotic agents, inhaled steroids, bronchodilators, mucolytics, cough preparations); diagnostic tests (chest X-rays, pulmonary function tests, sputum cultures, blood tests); referrals to respiratory consultants; emergency room visits; and hospital admissions (including length of stay). The information on changes in respiratory symptoms as recorded in the GPs files was often incomplete and therefore not used in the current evaluation. Separate contacts pertaining to one and the same exacerbation episode were marked as such by the reporting GP. Completeness of the reported contacts was verified by one of the investigators in a $10 \%(n=29)$ random sample of study subjects. In this sample, completeness of reporting turned out to be $100 \%$.

Spirometry was performed in a pulmonary function laboratory in all subjects following the European Respiratory Society (ERS) standards [27]. Following the protocol of the subsequent clinical trial [24], subjects were categorised according to the severity of airflow obstruction using criteria issued by the ERS (postbronchodilator $\mathrm{FEV}_{1}$ as percentage of predicted value $\geq 80 \%$ : no obstruction; $\geq 70 \%$ and $<80 \%$ : mild obstruction; $\geq 50 \%$ and $<70 \%$ : moderate obstruction; $<50 \%$ : severe obstruction) [26]. History of cigarette smoking was assessed by standardised interview and quantified as the number of packyears smoked.

\section{Cost calculations}

Use of healthcare resources was assessed by counting the number of units consumed. Subsequently, units were converted into monetary values. Drug prescriptions were converted to costs using the March 2000 table of the Royal Dutch Association for the Advancement of Pharmacy and included taxes and pharmacist fee. Cost of diagnostic tests were calculated using tariffs published by the Dutch Council of Health Insurances [28, 29]. Referrals to respiratory consultants, emergency visits, and hospital admissions were valued using results of a cost investigation from a secondary care population of patients with COPD [30]. The cost of referrals included the amount time spent by respiratory consultants and additional diagnostic procedures requested by the consultants. Costs were calculated as the cost per exacerbation episode as well as the annual exacerbation cost per subject. All cost are expressed in Euros. The year 2001 was taken as the index year for all cost, regardless of the calendar year in which an exacerbation had actually occurred.

\section{Analyses}

The SAS statistical software package (version 6.12 for Windows) was used for analysis. Differences in baseline characteristics between the categories of obstruction severity were analysed using analysis of variance (ANOVA) and $\chi^{2}$ tests. Mean and median annual exacerbation rates were calcu- 
lated for the four categories of obstruction severity and compared by ANOVA and Kruskal-Wallis tests. Differences in the occurrence of exacerbations by calendar month were analysed by $\chi^{2}$ test. Although the cost data distribution was skewed towards the left, $t$ test based $95 \%$ confidence intervals (95\% CIs) were calculated for total cost and separate cost components [31]. Differences in exacerbation related cost between obstruction severity subgroups were compared using ANOVA.

\section{Results}

\section{Study population}

Demographic and clinical characteristics of the study population are given in table 1. Age and packyears of smoking were highest for the subjects with more severe obstruction (both $\mathrm{p}<0.001$ ), whereas the body mass index (BMI) was lowest in the more severe subgroups $(\mathrm{p}=0.042)$. Reversibility as percentage of predicted $\mathrm{FEV}_{1}$ ranged from 5.5 (SD 6.2) percent in subjects without obstruction to 8.0 (SD 5.8) percent in subjects with moderate obstruction $(\mathrm{p}=0.044)$. Long-acting bronchodilators and inhaled steroids were more often prescribed in the more severely affected subgroups $(\mathrm{p}=0.003$ and $\mathrm{p}=0.019$, respectively). Comorbidity was present in 109 (38\%) subjects, hypertension being the most prevalent condition $(16 \%$ of all subjects) followed by ischemic heart disease $(15 \%)$, atherosclerosis $(11 \%)$, cardiac arrhythmia $(6 \%)$, and diabetes mellitus $(5 \%)$. Presence of relevant comorbid conditions did not differ between the categories of obstruction severity. Following the GOLD criteria, $14 \%$ of all patients had mild COPD (GOLD I), 48\% moderate COPD (GOLD II), and $16 \%$ severe COPD (GOLD III). $22 \%$ of

Table 1. - Demographic and clinical characteristics of the study population $(n=286)$ by severity of airflow obstruction $^{*}$ at the end of the 2-yr retrospective observation period. Values are means (standard deviation) unless stated otherwise

\begin{tabular}{|c|c|c|c|c|c|c|}
\hline & $\begin{array}{l}\text { Chronic } \\
\text { bronchitis }\end{array}$ & $\begin{array}{c}\text { Mild } \\
\text { obstruction }\end{array}$ & $\begin{array}{l}\text { Moderate } \\
\text { obstruction }\end{array}$ & $\begin{array}{c}\text { Severe } \\
\text { obstruction }\end{array}$ & Total & $\mathrm{p}^{\dagger}$ \\
\hline \multicolumn{7}{|l|}{ General characteristics } \\
\hline $\begin{array}{l}\mathrm{n}(\%) \\
\text { Age vears }\end{array}$ & $75(26)$ & $53(19)$ & $\begin{aligned} 114(40) \\
597(88)\end{aligned}$ & $\begin{array}{r}44(15) \\
643(9)\end{array}$ & $286(100)$ & \\
\hline Age, years & $56.6(9.8)$ & $57.6(9.6)$ & $59.7(8.8)$ & $64.3(9.2)$ & $59.2(9.6)$ & $<0.001$ \\
\hline Males (\%) & $54(72)$ & $32(60)$ & $90(69)$ & $32(73)$ & $209(73)$ & 0.097 \\
\hline Paid work (\%) & $32(43)$ & $15(28)$ & $36(32)$ & $6(14)$ & $89(31)$ & 0.011 \\
\hline Current smokers (\%) & $42(56)$ & $33(62)$ & $60(53)$ & $26(59)$ & $160(56)$ & 0.675 \\
\hline Cigarette smoke exposure, packyears & $25(14)$ & $24(14)$ & $29(20)$ & 37 (17) & $28(17)$ & $<0.001$ \\
\hline Body mass index, $\mathrm{kg} / \mathrm{m}^{2}$ & $26.8(4.5)$ & $27.6(5.1)$ & $25.8(3.7)$ & $25.5(4.6)$ & $26.4(4.4)$ & 0.042 \\
\hline \multicolumn{7}{|l|}{ Comorbid conditions (\%) } \\
\hline None & $53(71)$ & $31(58)$ & $73(64)$ & $20(45)$ & $177(62)$ & \\
\hline 1 & $12(20)$ & $12(23)$ & $24(21)$ & $15(34)$ & $66(23)$ & \\
\hline 2 or more & $7(9)$ & $10(19)$ & $17(15)$ & $9(21)$ & $43(15)$ & 0.181 \\
\hline \multicolumn{7}{|l|}{ Medication use } \\
\hline On inhaled corticosteroids (\%) & $26(35)$ & $27(51)$ & $51(45)$ & $28(64)$ & $132(46)$ & 0.019 \\
\hline \multicolumn{7}{|l|}{ Bronchodilator treatment (\%) } \\
\hline No bronchodilator & $30(40)$ & $10(19)$ & $24(21)$ & $5(11)$ & $69(24)$ & \\
\hline Short-acting & $37(49)$ & $34(64)$ & $63(55)$ & $26(59)$ & $160(56)$ & \\
\hline Long-acting $\ddagger$ & $8(11)$ & $9(17)$ & $27(24)$ & $13(30)$ & $57(20)$ & 0.003 \\
\hline \multicolumn{7}{|l|}{ Pulmonary function } \\
\hline Prebronchodilator $\mathrm{FEV}_{1}$, litres & $2.54(0.56)$ & $2.06(0.51)$ & $1.61(0.39)$ & $1.03(0.26)$ & $1.85(0.68)$ & $<0.001$ \\
\hline as $\%$ predicted & $81.6(8.7)$ & $68.1(7.5)$ & $51.9(8.8)$ & $36.4(7.4)$ & $60.3(17.8)$ & $<0.001$ \\
\hline Postbronchodilator $\mathrm{FEV}_{1}$, litres & $2.72(0.55)$ & $2.26(0.47)$ & $1.85(0.36)$ & $1.20(0.24)$ & $2.05(0.66)$ & $<0.001$ \\
\hline as $\%$ predicted & $87.1(6.1)$ & $74.9(3.1)$ & $59.8(5.6)$ & $42.2(5.7)$ & $67.1(16.2)$ & $<0.001$ \\
\hline Postbronchodilator FVC, litres & $3.95(0.97)$ & $3.36(0.85)$ & $3.21(0.82)$ & $2.45(0.56)$ & $3.32(0.96)$ & $<0.001$ \\
\hline as $\%$ predicted & $102.4(14.5)$ & $90.6(13.7)$ & $82.7(14.3)$ & $68.8(13.0)$ & $87.2(17.9)$ & $<0.001$ \\
\hline Postbronchodilator $\mathrm{FEV}_{1} / \mathrm{FVC}, \%$ & $69.9(7.6)$ & $68.4(9.1)$ & $59.1(10.3)$ & $50.4(11.8)$ & $62.3(11.9)$ & $<0.001$ \\
\hline $\mathrm{FEV}_{1}$ reversibility, \%§ & $5.5(6.2)$ & $6.8(8.0)$ & $8.0(5.8)$ & $5.9(4.3)$ & $6.8(6.2)$ & 0.044 \\
\hline \multicolumn{7}{|l|}{ Annual exacerbation rate } \\
\hline Mean rate & $0.75(0.63)$ & $0.85(0.76)$ & $0.97(0.88)$ & $0.89(0.78)$ & $0.88(0.79)$ & 0.303 \\
\hline Median rate (IQR) & $0.5(1.0)$ & $0.5(0.7)$ & $0.7(1.0)$ & $0.5(1.4)$ & $0.5(1.0)$ & 0.627 \\
\hline $\begin{array}{l}\text { * } \text { Chronic bronchitis: post-BD FEV } \\
70-80 \% \text { of predicted; moderate obs } \\
\dagger \quad \text { p-values indicate significance level } \\
+\quad \text { with or without additional short-act } \\
\S \text { difference in FEV1\% predicted be } \\
\text { IQR = interquartile range. }\end{array}$ & $\begin{array}{l}0 \% \text {, but } \mathrm{FEV}_{1} / \\
\text { uction: } \mathrm{FEV}_{1} \\
\text { of differences } \\
\mathrm{g} \text { bronchodila } \\
\text { een prebronch }\end{array}$ & $\begin{array}{l}\mathrm{VC}<88 \%(<8 \\
0-69 \% \text { of pre } \\
\text { etween obstr } \\
\text { or. } \\
\text { odilator and } \mathrm{p}\end{array}$ & $\begin{array}{l}\text { \% for women) } \\
\text { cted; severe o } \\
\text { tion severity } \\
\text { tbronchodilat }\end{array}$ & $\begin{array}{l}\text { f predicted val } \\
\text { truction: FEV } \\
\text { bgroups. } \\
\text { measurement }\end{array}$ & 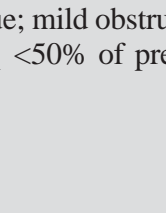 & $\begin{array}{l}\text { on: } \mathrm{FEV}_{1} \\
\text { cted [24]. }\end{array}$ \\
\hline
\end{tabular}


the study population consistent of current or former smokers with chronic bronchitis (GOLD 0), although their fulfillment of the studies' inclusion criteria indicated incipient lung function abnormality.

\section{Occurrence of exacerbations}

A total of 507 exacerbations comprising 732 GP contacts were reported for the 286 subjects studied. In 227 (45\%) exacerbations more than one contact with the GP had taken place. The number of GP contacts per exacerbation ranged from 1 to 7 , with an average of 1.5 (SD 0.9) contacts. 220 subjects $(77 \%)$ experienced at least one exacerbation during the two year period. Mean and median annual exacerbation rates did not differ between the severity subgroups (table 1). There was a dissimilar distribution in the occurrence of exacerbations throughout the year, with the majority of exacerbations reported in the months of October through to March (59\% of all exacerbations) and July ( $\chi^{2}$ test, $p=0.014$, figure 1$)$.

\section{Management of exacerbations}

A course of oral corticosteroids was prescribed in $26 \%$ of all exacerbations. Within the set of all reported exacerbations, the probability of an oral corticosteroid prescription increased roughly along with the severity of obstruction of the subject concerned (no obstruction: 0.18, mild: 0.28 ; moderate: 0.28 ; severe: $0.32 ; \chi^{2}$ test: $\left.\mathrm{p}=0.040\right)$. $47 \%$ of the exacerbations were treated with one or more antibiotic drugs, tetracyclines being the most frequently prescribed class of antibiotics (56\%), followed by penicillins $(27 \%)$, macrolides (12\%), sulfonamides (4\%), and quinolones (1\%). Unlike oral corticosteroid prescriptions, the probability of an antibiotic being prescribed did not increase as obstruction became more severe $\left(\chi^{2}\right.$ test, $\left.\mathrm{p}=0.747\right)$. Hospital admission was reported for only one exacerbation; one other exacerbation necessitated an emergency room visit. Spirometry was performed in $173(34 \%)$ of all exacerbations (94\% performed in general practice, $6 \%$ in a pulmonary function laboratory). Chest radiographs were requested in $63(12 \%)$ exacerbations, sputum cultures in $3(1 \%)$. Referral to a respiratory consultant was reported for $25(5 \%)$ exacerbations.

\section{Exacerbation related healthcare cost}

Table 2 shows the mean cost per exacerbation and the mean annual exacerbation cost per study subject. The total cost per exacerbation aggregated to $€ 66(95 \%$ CI 56, 76). The annual cost per subject attributable to exacerbations aggregated to $€ 59$ (95\% CI 48, 69). Exclusion of the hospital admission cost resulted in $€ 62$ (95\% CI 57, 67) per exacerbation, and $€ 51$ (95\% CI 44, 57) for the annual exacerbation cost. There was no significant association between the annual exacerbation cost and age (Spearman: $r=0.027, p=0.654$ ) or gender

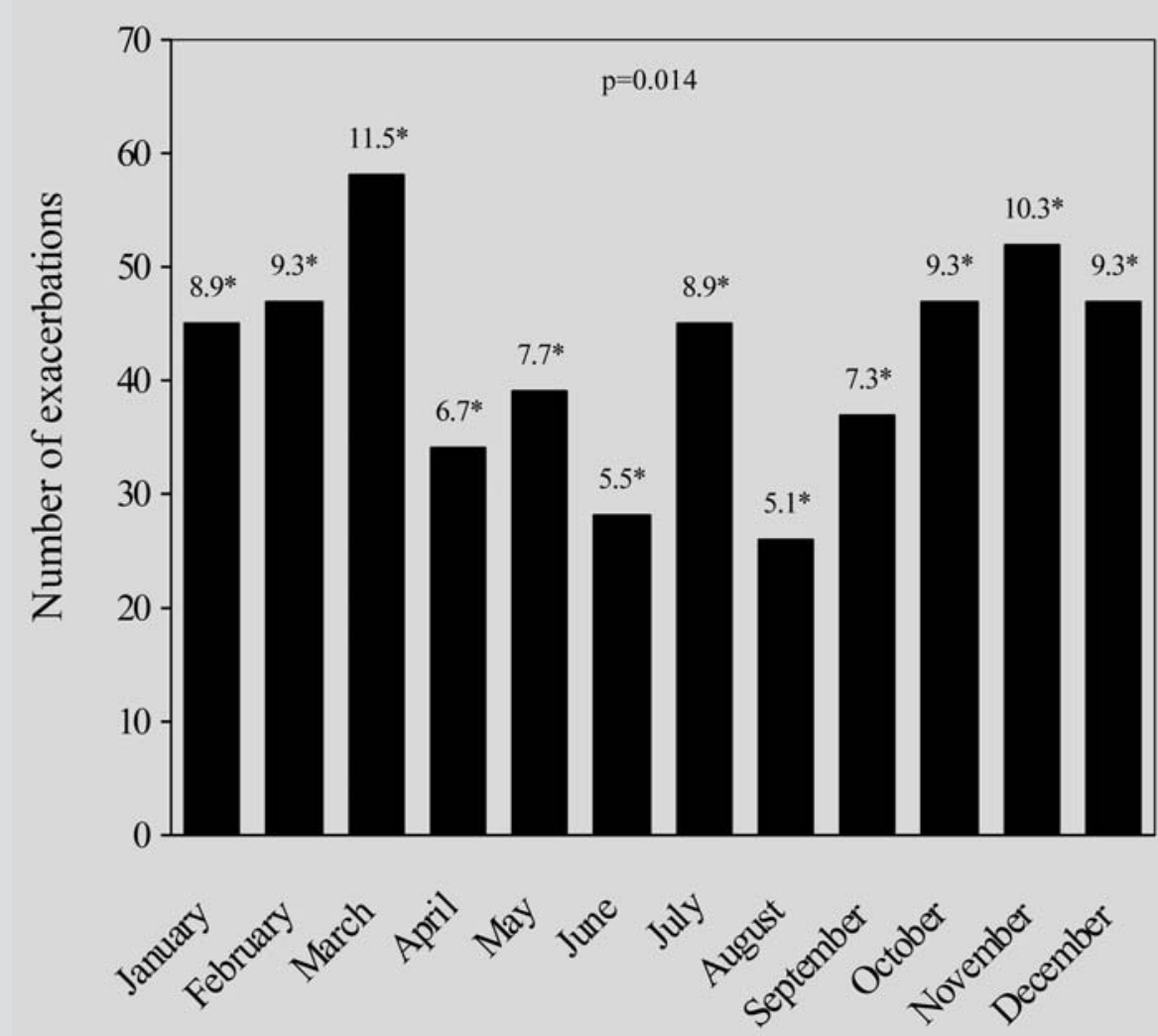

Fig. 1. - Occurrence of exacerbations by calender month in 286 subjects with COPD managed in general practice. The solid line represents the overall mean number of exacerbations per calender month, the figure above each bar is the proportion of all reported exacerbations. 
Table 2. - Mean $(95 \% \mathrm{Cl}$ ) healthcare cost (in Euros) for exacerbations, and mean annual cost attributable to exacerbations in subjects with COPD

\begin{tabular}{|c|c|c|c|}
\hline & $\begin{array}{l}\mathrm{n}^{\circ} \text { of } \\
\text { units }\end{array}$ & $\begin{array}{c}\text { Cost per } \\
\text { exacerbation } \\
(\mathrm{n}=507)\end{array}$ & $\begin{array}{l}\text { Annual exacerbation } \\
\text { cost per subject } \\
(n=286)\end{array}$ \\
\hline \multicolumn{4}{|l|}{ GP consultations } \\
\hline Office visits & 681 & $22.6(21.3,23.8)$ & $19.9(17.7,22.2)$ \\
\hline House calls & 35 & $1.7(1.0,2.5)$ & $1.5(0.8,2.2)$ \\
\hline Phone consultations & 16 & $0.2(0.1,0.3)$ & $0.2(0.1,0,3)$ \\
\hline Total GP consultation cost & & $24(23,26)$ & $22(19,24)$ \\
\hline \multicolumn{4}{|l|}{ Drug prescriptions } \\
\hline Antibiotic agents & 260 & $5.7(5.0,6.4)$ & $5.0(4.1,5.9)$ \\
\hline Oral corticosteroids & 149 & $1.6(1.4,1.9)$ & $1.4(1.1,1.8)$ \\
\hline Inhaled corticosteroids & $106^{*}$ & $7.1(5.4,8.7)$ & $6.1(4.3,7.9)$ \\
\hline Bronchodilators & $179 \dagger$ & $5.7(4.6,6.8)$ & $4.9(3.7,6.1)$ \\
\hline Other reliever medication & 17 & $0.4(0.2,0.7)$ & $0.4(0.2,0.6)$ \\
\hline Total drug prescription cost & & $20(18,23)$ & $18(15,21)$ \\
\hline \multicolumn{4}{|l|}{ Diagnostic procedures } \\
\hline Chest X-rays & 63 & $4.9(3.7,6.0)$ & $4.3(3.2,5.4)$ \\
\hline Lung function tests & 173 & $5.7(4.7,6.8)$ & $5.1(3.9,6.3)$ \\
\hline Sputum cultures & 3 & $0.1(0,0.2)$ & $0.0(0.0,0.1)$ \\
\hline Total diagnostic procedure cost & & $11(9,12)$ & $9(8,11)$ \\
\hline \multicolumn{4}{|l|}{ Secondary care } \\
\hline Consultation of respiratory consultant & $25 \S$ & $6.0(3.7,8.3)$ & $5.3(3,2,7.4)$ \\
\hline Emergency room visits & 111 & 0.3 & 0.6 \\
\hline Hospital admissions & $1 \&$ & 4.4 & 7.8 \\
\hline Total secondary care cost & & $11(2,20)$ & $10(2,17)$ \\
\hline \multicolumn{2}{|l|}{ Total healthcare cost } & $66(56,76)$ & $59(48,69)$ \\
\hline \multicolumn{4}{|c|}{ * $76(72 \%)$ new prescriptions, $30(28 \%)$ increased dosage. } \\
\hline \multicolumn{4}{|c|}{$130(73 \%)$ new prescriptions, $49(27 \%)$ increased dosage. } \\
\hline \multicolumn{4}{|c|}{$\S$ including cost of diagnostic procedures requested by the consultant. } \\
\hline \multicolumn{4}{|l|}{11 cost of the ER visit were $€ 161$. } \\
\hline$\&$ cost of the hospital admission were & & & \\
\hline
\end{tabular}

(Mann-Whitney U, $\mathrm{p}=0.492$ ). A consistent tendency towards increasing annual exacerbation cost within the more severely obstructed subgroups was observed (figure $2, p=0.024$, and $p=0.060$ with the hospital admission cost excluded). The increasing costs were merely due to more GP consultations, diagnostic procedures requested, and prescriptions for reliever medication.

\section{Discussion}

In this study we assessed the occurrence of exacerbations in a group of patients with COPD treated in Dutch general practice, and estimated the healthcare costs arising from these acute episodes. We observed that COPD patients with more severe airflow obstruction did not experience more frequent exacerbations, but once an exacerbation had occurred the associated healthcare costs became higher as the obstruction was more severe. The composition of the patient population studied was rather representative of the Netherlands, although patients with mild COPD (GOLD I) were somewhat underrepresented (14\%, against an estimated $27 \%$ in the Dutch general practice population) [23].

\section{Comparison with previous studies}

Our results indicate that the healthcare cost induced by exacerbations of COPD in (Dutch) primary care may depend on the severity of airflow obstruction, whereas the occurrence rate of exacerbations itself does not seem to do so. The latter observation is in contrast with a previous COPD study conducted in the Spanish primary care setting, in which an association was found between the severity of airflow obstruction and the risk of frequent exacerbations [32]. A possible explanation for these contradictory findings may be that the Spanish study did not include patients with an $\mathrm{FEV}_{1} \%$ predicted $>80 \%$ and/or $\mathrm{FEV}_{1} / \mathrm{FVC}$ ratio $>70 \%$, which means that patients with mild COPD (GOLD I) or subjects at risk of COPD (GOLD 0) were not included like in our study. Overall, the Spanish patient sample [32] suffered - on average - from more severe COPD, was about a decade older than our sample (68 versus 59 years), had substantially higher number of packyears (41 versus 28), and showed a substantially higher annual exacerbation rate (median of 2 exacerbations/year versus 0.5 exacerbations/year). These, alongside other differences (for instance the definition of an 


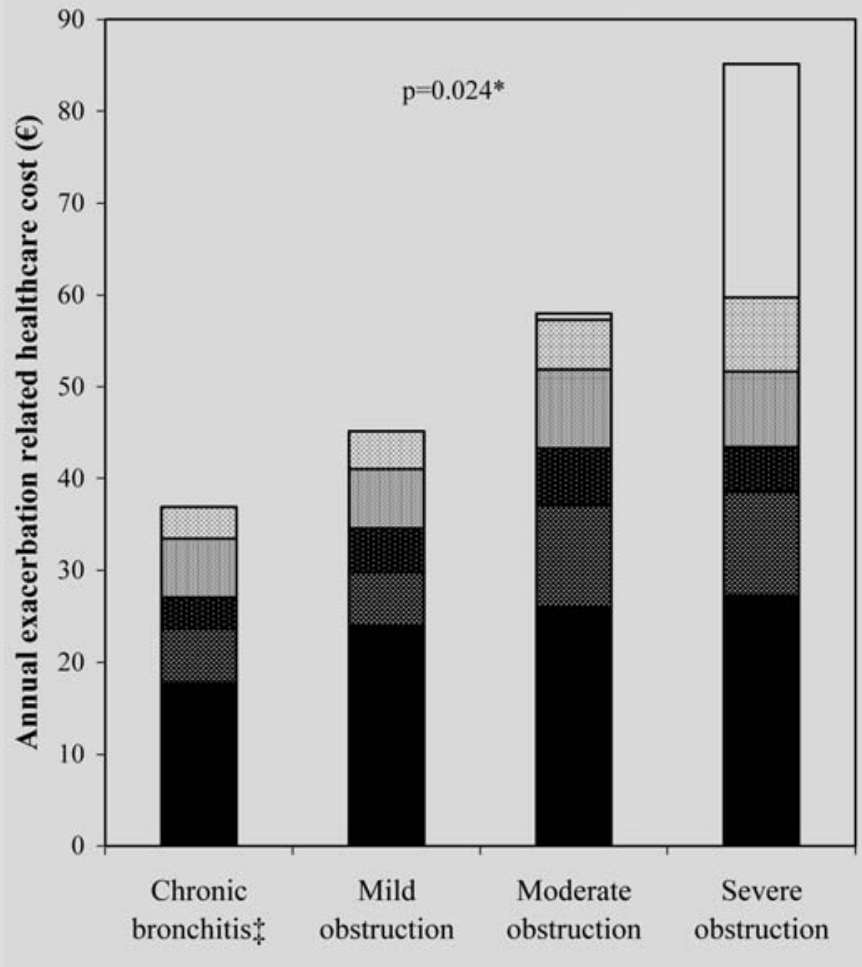

$\square$ Hospitalizations

$\square$ Reliever medication $\dagger$

Anti-inflammatory

Antibiotic agents

Diagnostic procedures

Physician time

pen.060 with the hospital cost excluded. bronchodilators, mucolytics, and cough preparations.

$¥$ without obstruction, i.e. post-bronchodilator $\mathrm{FEV}_{1}>80 \%$ of predicted value.

Fig. 2. - Distribution of exacerbation related annual healthcare cost by subgroup of severity of airflow obstruction.

exacerbation) between the studies as well as differences between the healthcare systems in the Netherlands and Spain should be taken into account when comparing the two studies. On the other hand, our findings are in line with studies performed in a UK secondary care setting, in which patients with frequent or infrequent exacerbations were indistinguishable in terms of lung function [33]. In the current study, the patients with more severe obstruction did not so much experience more exacerbations but once they suffered from one, the healthcare costs involved were higher than in those with less severe obstruction. The higher costs were caused primarily by more consultations, diagnostic tests, and prescription of reliever medication (i.e., bronchodilators, mucolytics, and cough preparations) in the GPs management of exacerbations. A relationship between $\mathrm{FEV}_{1}$ impairment and increased drug prescription in stable COPD has been reported previously [11], but our data suggests that this relationship may also apply to the primary care cost of treating acute exacerbations of the disease. Recent studies from Sweden and France confirm our observations [34, 35].

Studies in patients with COPD treated in secondary care have shown that the treatment cost of exacerbations are largely due to hospitalisations, emergency room visits, and domestic oxygen therapy $[10,19]$. Compared to these studies the exacerbation related costs in our study were rather low. This is easily explained by the fact that among all 507 reported exacerbations only one hospital ad- mission and one emergency room visit had occurred. This further demonstrates the typical primary care nature of our study population: in the Netherlands, patients treated by GPs generally suffer from less severe disease and, consequently, require less intensive medical attention in case of an exacerbation compared to patients treated by secondary care chest physicians. The Confronting COPD survey has also demonstrated that the rate of unscheduled healthcare contacts is relatively low in the Netherlands [36].

\section{Patient sample, definition of exacerbations, and cost estimations}

One of the main reasons for Dutch GPs to refer patients with COPD to a chest physician appears to be the occurrence of recurrent or unresolving exacerbations [37]. Once a patient with COPD has been admitted to hospital because of a (severe) exacerbation, it is rather common practice that a chest physician temporarily or permanently takes over the patients' treatment after discharge from hospital. Because of this, patients who were treated by chest physicians were less likely to be invited by the GPs for participation in the upcoming trial and because patients with a predicted $\mathrm{FEV}_{1}<40 \%$ of were excluded, those with frequent or severe exacerbations were probably underrepresented in our study population.

In order to avoid being forced to choose between existing definitions for exacerbations be- 
forehand, we instructed the GPs to report any contact in which study subjects sought medical attention because of acute deterioration of their respiratory condition. Because of the differing definitions for exacerbation used in the literature [38], direct comparison between the exacerbation rate observed in our study with rates reported from previous studies is impeded. One method is to use prescriptions for courses of oral corticosteroids and antibiotics as a proxy measure for exacerbations [39]. However, had we only used this proxy definition in the current study, we would have missed a substantial number of exacerbations, as in only a quarter of all episodes an oral corticosteroid was prescribed. With the addition of antibiotic prescriptions to the proxy definition based oral corticosteroid prescriptions alone [13], $59 \%$ of all episodes would have been detected. Still, a substantial part $(41 \%)$ of all episodes was managed without a course of oral corticosteroids or antibiotics, but merely with a -transient- increase of the dosage of inhaled corticosteroids and/or bronchodilators, or prescription of other reliever medication (i.e., mucolytics, cough preparations).

In the Netherlands each inhabitant can only be registered at one single general practice. Because of this, it is unlikely that we missed many exacerbations by using GP records as the only data source. However, we can only speculate on the number of exacerbations which were 'self-managed' by patients and were therefore not included in the GPs' medical records. It has been reported that patients with COPD may seek medical attention in only $50 \%$ of exacerbations [14]. From a healthcare expenditure perspective these events are irrelevant as they do not result in any medical costs. However, the 'productivity' costs due to work absenteeism or incapacity for work may be relevant in these episodes. Due to the retrospective nature of our method for data collection we were not able to include the exacerbation related productivity cost in our calculations.

In conclusion, in the typical primary care population of patients with COPD studied we observed that patients with increasing severity of airflow obstruction experienced more expensive, but not more frequent exacerbations. The additional costs were mainly due to more physician consultations, requested diagnostic tests, and new prescriptions or adjustments of existing medication for relieve of respiratory symptoms. Although the probability of an oral corticosteroid prescription increased roughly along with the severity of airflow obstruction, a substantial number of exacerbations were not treated with either oral corticosteroids or antibiotics, which makes this healthcare utilisation based proxy measure a rather unreliable instrument for assessing exacerbation occurrence rates in primary care COPD studies.

Acknowledgement: The authors wish to acknowledge all patients and general practitioners who contributed to this study.

\section{References}

1. Global Initiative for Chronic Obstructive Lung Disease $^{\mathrm{TM}}$ (GOLD). www.goldcopd.com. Date accessed: 20 February 2006.

2. Strassels SA. Economic consequences of chronic obstructive pulmonary disease. Curr Opin Pulm Med 1999; 5: 100-104.

3. Rossi A, Confalonieri M. Burden of chronic obstructive pulmonary disease. Lancet 2000; 356 Suppl: s56.

4. Wedzicha JA. Exacerbations: etiology and pathophysiologic mechanisms. Chest 2002; 121 (5 Suppl): 136S$141 \mathrm{~S}$.

5. Seemungal TAR, Donaldson GC, Paul EA, et al. Effect of exacerbation on quality of life in patients with chronic obstructive pulmonary disease. Am J Respir Crit Care Med 1998; 157: 1418-1422.

6. Osman LM, Godden DJ, Friend JAR, et al. Quality of life and hospital re-admission in patients with chronic obstructive pulmonary disease. Thorax 1997; 52: 67-71.

7. Nicolson P, Anderson P. The patient's burden: physical and psychological effects of acute exacerbations of chronic bronchitis. J Antimicrob Chemother 2000; 45: 25-32.

8. Dewan NA, Rafique S, Kanwar B, et al. Acute exacerbation of COPD: factors associated with poor treatment outcome. Chest 2000; 117: 662-671.

9. Niewoehner DE, Collins D, Erbland ML. Relation of FEV (1) to clinical outcomes during exacerbations of chronic obstructive pulmonary disease. Department of Veterans Affairs Cooperative Study Group. Am J Respir Crit Care Med 2000; 161: 1201-1205.

10. Connors AF, Dawson NVD, Thomas C, et al. Outcomes following acute exacerbations of severe chronic obstructive lung disease. The SUPPORT investigators (Study to Understand Prognoses and Preferences for Outcomes and Risks of Treatments). Am J Respir Crit Care Med 1996; 154: 959-967.

11. Donaldson GC, Seemungal TA, Bhowmik A, et al. Relationship between exacerbation frequency and lung function decline in chronic obstructive pulmonary disease. Thorax 2002; 57: 847-852.

12. Miravitlles M, Mayordomo C, Artes M, et al. Treatment of chronic obstructive pulmonary disease and its exacerbations in general practice. Respir Med 1999; 93: 173-179.

13. Burge PS, Calverley PMA, Jones PW, et al. Randomised, double blind placebo controlled study of fluticasone propionate in patiens with moderate to severe chronic obstructive pulmonary disease: the ISOLDE trial. BMJ 2000; 320: 1297-1303.

14. Seemungal TA, Donaldson GC, Bhowmik A, et al. Time course and recovery of exacerbations in patients with chronic obstructive pulmonary disease. Am J Respir Crit Care Med 2000; 161: 1608-1613.

15. Ball P, Harris JM, Lowson D, et al. Acute infective exacerbations of chronic bronchitis. $Q J$ Med 1995; 88: 61-68.

16. Renkema TEJ, Schouten PJ, Koeter GH, et al. Effects of long-term treatment with corticosteroids in COPD. Chest 1996; 109: 1156-1162.

17. Vincken W, van Noord JA, Greefhorst AP, et al. Improved health outcomes in patients with COPD during 1 yr's treatment with tiotropium. Eur Respir J 2002; 19: 209-216.

18. Casaburi R, Mahler DA, Jones PW, et al. A long-term evaluation of once-daily inhaled tiotropium in chronic obstructive pulmonary disease. Eur Respir J 2002; 19: 217-224.

19. Niederman MS, McCombs JS, Unger AN, et al. Treatment cost of acute exacerbations of chronic bronchitis. Clin Ther 1999; 21: 576-591. 
20. Miravitlles M, Murio C, Guerrero T, et al. Pharmacoeconomic evaluation of acute exacerbations of chronic bronchitis and COPD. Chest 2002; 121: 1449-1455.

21. Miravitlles M, Murio C, Guerrero T, et al. Costs of chronic bronchitis and COPD: a 1-year follow-up study. Chest 2003; 123: 784-791.

22. Gibson PG, Wlodarczyk JH, Wilson AJ, et al. Severe exacerbation of chronic obstructive airways disease: Health resource use in general practice and hospital. $J$ Qual Clin Practice 1998; 18: 125-133.

23. Hoogendoorn M, Feenstra TL, Schermer TR, Hesselink AE, Rutten-van Molken MP. Severity distribution of chronic obstructive pulmonary disease (COPD) in Dutch general practice. Respir Med 2006; 100: 83-6.

24. Chavannes NH, Schermer TRJ, Wouters EF, et al. Treatment of COPD in general practice: the COOPT study. Eur Respir J 2001; 18 Suppl 33: 348s.

25. ICPC International Classification of Primary Care. Lamberts $\mathrm{H}$ and Wood M, editors. Oxford, Oxford University Press, 1987.

26. Siafakas NM, Vermeire P, Pride NB, et al. Optimal assessment and management of chronic obstructive pulmonary disease (COPD). Eur Respir J 1995; 8: 1398-1420.

27. Quanjer PH, Tammeling GJ, Cotes JE, et al. Lung volumes and forced ventilatory flows. Report Working Party Standardization of Lung Function Tests, European Community for Steel and Coal. Official Statement of the European Respiratory Society. Eur Respir J Suppl 1993; 16: 5-40.

28. Diagnostic Compass 1999/2000. [Information on additional diagnostic procedures.] Amstelveen: Dutch Council of Health Insurances, 1999.

29. Pharmacotherapeutic Compass 2000/2001 [Information on pharmaceutical products]. Amstelveen: Dutch Council of Health Insurances, 2000
30. Decramer M, Dekhuijzen PNR, Troosters T, et al. The Bronchitis Randomized On NAC Cost-Utility Study (BRONCUS): hypothesis and design. Eur Respir $J$ 2001; 17: 329-336.

31. Thompson SG, Barber JA. How should cost data in pragmatic randomised trials be analysed? BMJ 2000; 320: $1197-1200$.

32. Miravitlles M, Guerrero T, Mayordomo C, et al. Factors associated with increased risk of exacerbation and hospital admission in a cohort of ambulatory COPD patients: A multiple logicstic regression analysis. Respiration 2000; 67: 495-501.

33. Gompertz S, Bayley DL, Hill SL, et al. Relationship between airway inflammation and the frequency of exacerbations in patients with smoking related COPD. Tho$\operatorname{rax} 2001$; 56: 36-41.

34. Detournay B, Pribil C, Fournier M, et al. The SCOPE study: health-care consumption related to patients with chronic obstructive pulmonary disease in France. Value Health 2004; 7: 168-74.

35. Andersson F, Borg S, Jansson SA, et al. The costs of exacerbations in chronic obstructive pulmonary disease (COPD). Respir Med 2002; 96: 700-8.

36. Wouters EF. The burden of COPD in The Netherlands: results from the Confronting COPD survey. Respir Med 2003; 97 Suppl C: S51-S59.

37. Schermer T, Smeenk F, van Weel C. Referral and consultation in asthma and COPD: an exploration of pulmonologists' views. Neth J Med 2003; 61: 71-81.

38. Burge S, Wedzicha JA. COPD exacerbations: definitions and classifications. Eur Respir J Suppl 2003; 41: 46s-53s.

39. Pauwels RA, Lofdahl CG, Laitinen LA, et al. Longterm treatment with inhaled budesonide in persons with mild chronic obstructive pulmonary disease who continue smoking. New Engl J Med 1999; 340: 1948-1953.

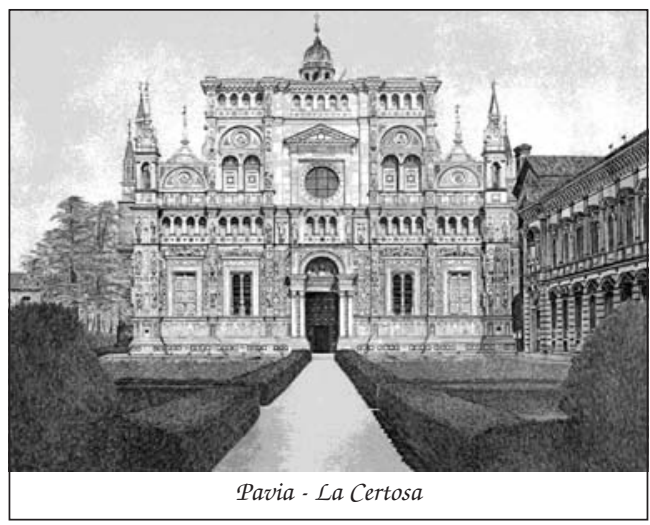

